\title{
Polarizabilities of Sc and Ti atoms and dispersion coefficients for their interaction with helium atoms
}

\section{Citation}

Chu, Xi, Alexander Dalgarno, and Gerrit C. Groenenboom. 2005. "Polarizabilities of Sc and Ti Atoms and Dispersion Coefficients for Their Interaction with Helium Atoms." Physical Review A 72 (3) (September 7). doi:10.1103/physreva.72.032703.

\section{Published Version}

doi:doi.org/10.1103/PhysRevA.72.032703

\section{Permanent link}

http://nrs.harvard.edu/urn-3:HUL.InstRepos:30403729

\section{Terms of Use}

This article was downloaded from Harvard University's DASH repository, and is made available under the terms and conditions applicable to Other Posted Material, as set forth at http:// nrs.harvard.edu/urn-3:HUL.InstRepos:dash.current.terms-of-use\#LAA

\section{Share Your Story}

The Harvard community has made this article openly available.

Please share how this access benefits you. Submit a story.

Accessibility 


\title{
Polarizabilities of Sc and Ti atoms and dispersion coefficients for their interaction with helium atoms
}

\author{
Xi Chu and Alexander Dalgarno \\ ITAMP, Harvard-Smithsonian Center for Astrophysics, 60 Garden Street, Cambridge, Massachusetts 02138, USA \\ Gerrit C. Groenenboom \\ Institute of Theoretical Chemistry, University of Nijmegen, Toernooiveld 1, 6525ED, Nijmegen, The Netherlands
}

(Received 11 June 2005; published 7 September 2005)

\begin{abstract}
The dynamic scalar and tensor polarizabilities of Sc and $\mathrm{Ti}$ are computed with time-dependent density functional theory. These polarizabilities are used to compute the isotropic and anisotropic dispersion interactions in ScHe and TiHe. We find $C_{6,0}(\mathrm{ScHe})=30.00, C_{6,2}(\mathrm{ScHe})=-1.63, C_{6,0}(\mathrm{TiHe})=28.40$, and $C_{6,2}(\mathrm{TiHe})$ $=-0.90\left(E_{h} a_{0}^{6}\right)$. We show that an estimate of the dispersion anisotropy based on static polarizabilities results in an overestimation by a factor of 2 .
\end{abstract}

DOI: $10.1103 /$ PhysRevA.72.032703

PACS number(s): 34.20.-b, 32.10.-f

\section{INTRODUCTION}

Experiments [1] have demonstrated that the cross sections for angular momentum transfer in collisions of ${ }^{3} \mathrm{He}$ atoms with rare earth gas atoms are small so that rare earth gas atoms can be cooled and trapped in a helium buffer gas. The ratio of the elastic and inelastic cross sections for $\mathrm{Sc}\left(3 d 4 s^{2}{ }^{2} \mathrm{D}\right)$ and $\mathrm{Ti}\left(3 d^{2} 4 s^{2}{ }^{3} \mathrm{~F}\right)$ atoms have been measured and are large [2]. The inelastic cross sections are controlled by the differences between the potential energy curves of the molecular symmetries populated by the approach of the complex atoms and helium $[3,4]$. The differences are small due to the shielding effects of the outermost shell of $4 s$ electrons which diminish the anisotropy of the interactions between $\mathrm{Sc}$ and $\mathrm{Ti}$ and ${ }^{3} \mathrm{He}$, as made evident by refined quantum chemistry calculations $[4,5]$. The calculations were carried out at intermediate and short internuclear distances. They lose accuracy at large distances where the interaction is the long range van der Waals interaction to which the cross sections are sensitive. Calculations of the van der Waals or dispersion coefficients for the interactions of complex atoms can be carried out to high precision $[6,7]$ and in themselves provide an assessment of the likelihood that specific atoms can be cooled in magnetic traps. We present detailed estimates of the leading dispersion coefficients for the individual symmetry states of the molecules $\mathrm{ScHe}$ and TiHe.

\section{THEORY}

The second order interaction between an open-shell atom with total orbital angular momentum $L$ and a $S$-state atom such as helium can be represented by an effective interaction potential $\hat{V}$ acting in the $2 L+1$ dimensional space $\{|L M\rangle, M$ $=-L, \ldots, L\}$, where $M$ is the projection of the angular momentum on the internuclear axis. The effective interaction potential $\hat{V}(R, \theta)$ is a function of the internuclear distance $R$ and the angle $\theta$ that specifies the orientation of the nonspherical atom with respect to the internuclear axis [8-12]. We expand $\hat{V}$ in Legendre polynomials $P_{K}(\cos \theta)$,

$$
\hat{V}(R, \theta)=\sum_{K} v_{K}(R) P_{K}(\cos \theta),
$$

where $R$ is the internuclear distance and $\theta$ is the polar angle of the effective electron. The molecular potentials $V_{M}(R)$ are related to the effective potential through

$$
V_{M}(R)=\langle L M|\hat{V}| L M\rangle=\sum_{K} v_{K}(R)\langle L M K 0 \mid L M\rangle\langle L 0 K 0 \mid L 0\rangle .
$$

The potentials $V_{M}$ are only labeled by $M$ because this is a good molecular quantum number; $M=0,1,2$, and 3 corresponds to $\Sigma, \Pi, \Delta$, and $\Phi$ molecular states. The expansion coefficients $v_{K}$ can be obtained from the molecular potentials by inverting this equation [9],

$$
v_{K}(R)=\frac{[K]}{[L]} \sum_{M=-L}^{L} \frac{\langle L M K 0 \mid L M\rangle}{\langle L 0 K 0 \mid L 0\rangle} V_{M}(R),
$$

where we used the orthogonality relation of Clebsch-Gordan coefficients,

$$
\sum_{M=-L}^{L}\langle L M K 0 \mid L M\rangle^{2}=\frac{[L]}{[K]}
$$

and $[X]=2 X+1$. At large distances $R$ the leading term in the potential is the dispersion interaction

$$
V_{M}(R) \simeq-\frac{C_{6}(L, M)}{R^{6}} .
$$

The $C_{6}$ coefficients may be calculated as [13]

$$
\begin{aligned}
C_{6}(L, M)= & \frac{2}{\pi} \int_{0}^{\infty} \alpha_{z z}(L, M ; i \omega) \bar{\alpha}_{\mathrm{He}}(i \omega) d \omega \\
& +\frac{1}{\pi} \int_{0}^{\infty} \alpha_{x x}(L, M ; i \omega) \bar{\alpha}_{\mathrm{He}}(i \omega) d \omega .
\end{aligned}
$$

The dynamic dipole-dipole polarizabilities at imaginary fre- 
quency $i \omega$ of state $|L M\rangle$ with energy $E_{L}, \alpha_{u u}(L, M ; i \omega)$, for $u \in\{x, y, z\}$, in atomic units, are given by

$$
\alpha_{u u}(L, M ; i \omega)=2 \sum_{\gamma M_{\gamma}} \frac{\left(E_{\gamma}-E_{L}\right)\left|\left\langle L M\left|\hat{\mu}_{u}\right| \gamma L_{\gamma} M_{\gamma}\right\rangle\right|^{2}}{\left(E_{\gamma}-E_{L}\right)^{2}+\omega^{2}},
$$

where $\hat{\mu}_{u}$ are the Cartesian components of the electric dipole operator and $E_{\gamma}$ is the energy of the excited electronic state $\left|\gamma L_{\gamma} M_{\gamma}\right\rangle$. For $u=z$ the summation involves only $M_{\gamma}=M$ and for $u=x$ we have $M_{\gamma}=M \pm 1$. The $z$ component refers to the internuclear axis so we define $\alpha_{\|}=\alpha_{z z}$ and $\alpha_{\perp}=\alpha_{x x}=\alpha_{y y}$. The $2(2 L+1)$ polarizabilities can be expressed in terms of the scalar polarizability $\alpha_{0}(L ; i \omega)$ and the tensor polarizability $[14,15] \alpha_{2}(L ; i \omega)$ (dropping the $\omega$ dependence for compactness),

$$
\begin{aligned}
\alpha_{\|}(L, M) & =\alpha_{0}(L)+\alpha_{2}(L) \frac{\langle L M 20 \mid L M\rangle}{\langle L L 20 \mid L L\rangle} \\
& =\alpha_{0}(L)+\alpha_{2}(L) \frac{3 M^{2}-L(L+1)}{L(2 L-1)}, \\
\alpha_{\perp}(L, M) & =\alpha_{0}(L)-\frac{1}{2} \alpha_{2}(L) \frac{3 M^{2}-L(L+1)}{L(2 L-1)},
\end{aligned}
$$

where the tensor polarizability is defined such that for $L$ $=M, \alpha_{\|}(L, L)=\alpha_{0}(L)+\alpha_{2}(L)$. For completeness we derive these expressions in the Appendix, where we also give the relation between the spherical and Cartesian components of the polarizabilities. From Eqs. (8) and (9) we see that all polarizabilities can be found if the parallel and perpendicular components of the polarizability for a single $|L M\rangle$ state are known,

$$
\begin{gathered}
\bar{\alpha}(L) \equiv \frac{1}{3} \alpha_{\|}(L, M)+\frac{2}{3} \alpha_{\perp}(L, M)=\alpha_{0}(L), \\
\Delta \alpha(L, M) \equiv \alpha_{\|}(L, M)-\alpha_{\perp}(L, M)=\frac{3}{2} \alpha_{2}(L) \frac{3 M^{2}-L(L+1)}{L(2 L-1)} .
\end{gathered}
$$

Alternatively, we may use Eq. (4) to derive

$$
\alpha_{K}(L)=\frac{[K]}{[L]}\langle L L K 0 \mid L L\rangle \sum_{M=-L}^{L}\langle L M K 0 \mid L M\rangle \alpha_{\|}(L, M)
$$

or, explicitly

$$
\begin{aligned}
& \alpha_{0}(L)=[L]^{-1} \sum_{M=-L}^{L} \alpha_{\|}(L, M), \\
& \alpha_{2}(L)= \frac{5}{(L+1)(2 L+1)(2 L+3)} \\
& \times \sum_{M=-L}^{L}\left[3 M^{2}-L(L+1)\right] \alpha_{\|}(L, M) .
\end{aligned}
$$

Equation (13) also holds when we replace $\alpha_{\|}$by $\alpha_{\perp}$ and in Eq. (14) we may replace $\alpha_{\|}$by $-2 \alpha_{\perp}$ [see Eqs. (8) and (9)]. From Eq. (13) the scalar polarizabilities for $P, D$, and $F$ state atoms are

$$
\begin{gathered}
\alpha_{0}(P)=\left[\alpha_{\|}(1,0)+2 \alpha_{\|}(1,1)\right] / 3, \\
\alpha_{0}(D)=\left[\alpha_{\|}(2,0)+2 \alpha_{\|}(2,1)+2 \alpha_{\|}(2,2)\right] / 5, \\
\alpha_{0}(F)=\left[\alpha_{\|}(3,0)+2 \alpha_{\|}(3,1)+2 \alpha_{\|}(3,2)+2 \alpha_{\|}(3,3)\right] / 7
\end{gathered}
$$

and from Eq. (14) we find the tensor polarizabilities,

$$
\begin{gathered}
\alpha_{2}(P)=\left[\alpha_{\|}(1,1)-\alpha_{\|}(1,0)\right] / 3, \\
\alpha_{2}(D)=\left[-2 \alpha_{\|}(2,0)-2 \alpha_{\|}(2,1)+4 \alpha_{\|}(2,2)\right] / 7, \\
\alpha_{2}(F)=\left[-10 \alpha_{\|}(3,0)-15 \alpha_{\|}(3,1)+25 \alpha_{\|}(3,3)\right] / 42 .
\end{gathered}
$$

In analogy to Eq. (3) we define the scalar $(K=0)$ and tensor $(K>0)$ components of the dispersion coefficients

$$
C_{6, K}(L)=\frac{[K]}{[L]} \sum_{M=-L}^{L} \frac{\langle L M K 0 \mid L M\rangle}{\langle L 0 K 0 \mid L 0\rangle} C_{6}(L, M) .
$$

Substituting Eqs. (6), (8), and (9) into Eq. (17) shows that $C_{6,0}(L)$ depends on $\alpha_{0}(L ; i \omega)$,

$$
C_{6,0}(L)=\frac{3}{\pi} \int_{0}^{\infty} \alpha_{0}(L ; i \omega) \bar{\alpha}_{\mathrm{He}}(i \omega) d \omega
$$

and that $C_{6,2}(L)$ depends on $\alpha_{2}(L ; i \omega)$

$$
C_{6,2}(L)=-\frac{3(2 L+3)}{2 \pi L} \int_{0}^{\infty} \alpha_{2}(L ; i \omega) \bar{\alpha}_{\mathrm{He}}(i \omega) d \omega
$$

and all other $C_{6, K}$ coefficients should be zero. Hence we can invert Eq. (17) [or use Eq. (2)] to express all dispersion coefficients as

$$
C_{6}(L, M)=C_{6,0}(L)-\frac{3 M^{2}-L(L+1)}{(2 L-1)(2 L+3)} C_{6,2}(L) .
$$

If the ratio $\alpha_{2}(i \omega) / \alpha_{0}(i \omega)$ is assumed to be independent of $\omega$ then we can estimate the anisotropy from the ratio of Eqs. (18) and (19)

$$
\frac{C_{6,2}(L)}{C_{6,0}(L)} \approx-\frac{2 L+3}{2 L} \frac{\alpha_{2}(L)}{\alpha_{0}(L)}
$$

\section{COMPUTATIONAL METHOD}

The dynamic polarizabilities $\alpha_{\|, \perp}(L, M ; i \omega)$ of Sc and Ti were computed using a linear response time-dependent density functional theory (TDDFT). A detailed description of the method is given in Refs. [6,7]. The TDDFT method has with few exceptions yielded polarizabilities good to within an uncertainty of about 5\%. The TDDFT polarizabilities are exact in the limit of high frequencies, varying asymptotically as $N / \omega^{2}$, where $N$ is the number of electrons in the system [6].

The DFT based approach applies to cases where a single determinant can be found as a reference to describe the 
TABLE I. The scalar and tensor static dipole polarizabilities of Sc and Ti (in $\left.a_{0}^{3}\right)$.

\begin{tabular}{ccccccc}
\hline \hline & \multicolumn{3}{c}{$\mathrm{Sc}$} & & \multicolumn{2}{c}{$\mathrm{Ti}$} \\
\cline { 2 - 3 } \cline { 5 - 6 } \cline { 5 - 6 } & \multicolumn{1}{c}{$\alpha_{0}$} & $\alpha_{2}$ & & $\alpha_{0}$ & $\alpha_{2}$ \\
\hline This paper & 105.88 & 6.31 & & 94.69 & 3.97 \\
Ref. [17] & 150.57 & 5.36 & & 129.34 & 2.17 \\
Ref. [18] & 107 & & & 91.4 & \\
Ref. [20] & 123.60 & 5.38 & & 104.05 & 2.06 \\
Ref. [20] & 121.22 & 5.24 & & 101.57 & 2.01 \\
\hline \hline
\end{tabular}

${ }^{\mathrm{a}}$ Nonrelativistic.

${ }^{\mathrm{b}}$ With Douglas-Kroll relativistic corrections.

atomic state. For $\operatorname{Sc}\left(3 d 4 s^{2}{ }^{2} \mathrm{D}\right)$ we can compute all six polarizabilities $(|M|=0,1,2, \|, \perp)$ but for $\operatorname{Ti}\left(3 d^{2} 4 s^{2}{ }^{3} \mathrm{~F}\right)$ only $M=2$ and $M=3$ are qualified and we compute four polarizabilities. Theoretically we can obtain the scalar and tensor polarizabilities, and hence all others, by solving the linear Eqs. (8) and/or (9) using only two independent polarizabilities $\alpha_{\|, \perp}(L, M ; i \omega)$. Instead we use a linear least squares fit to obtain the best possible $\alpha_{0}(L)$ and $\alpha_{2}(L)$ and we use the deviations from Eqs. (8) and/or (9) to check the internal consistency of the method and to obtain an error estimate.

The dynamic polarizability of helium has been determined to high precision by a large scale variational calculation [16]. We use it together with the scalar and tensor polarizabilities obtained from the least squares fit to the TDDFT results to compute the dispersion coefficients $C_{6,0}(L)$ [Eq. (18)] and $C_{6,2}(L)$ [Eq. (19)].

\section{RESULTS AND DISCUSSION}

In Table I we present the scalar and tensor static polarizabilities of Sc and Ti obtained from the least squares fits. The results for Ti were obtained from a fit to $\alpha_{\|}(3,2)=94.78$, $\alpha_{\|}(3,3)=98.62, \alpha_{\perp}(3,2)=94.78$, and $\alpha_{\perp}(3,3)=92.63 a_{0}^{3}$. The six static polarizabilities $\left[\alpha_{\|, \perp}(L, M)\right]$ computed for Sc are shown in Fig. 1. This figure also shows that the results for $\mathrm{Sc}$ are represented quite well by the scalar and tensor polariz-

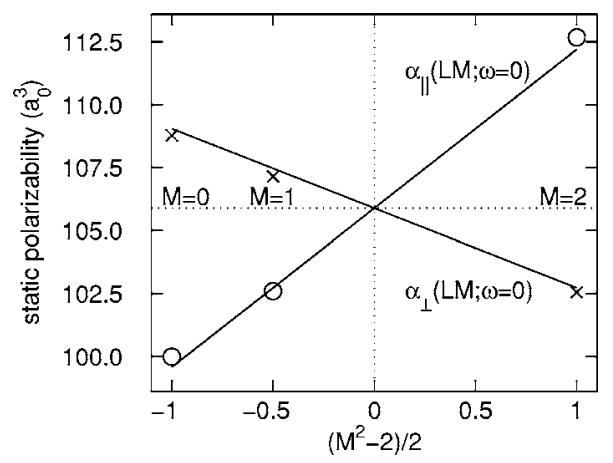

FIG. 1. The static polarizabilities $\alpha_{\|}(L M)$ (the circles) and $\alpha_{\perp}(L M)$ (the crosses) of Sc for $M=0,1$, and 2. The solid lines represent the result of a linear least squares fit using Eqs. (8) and (9).
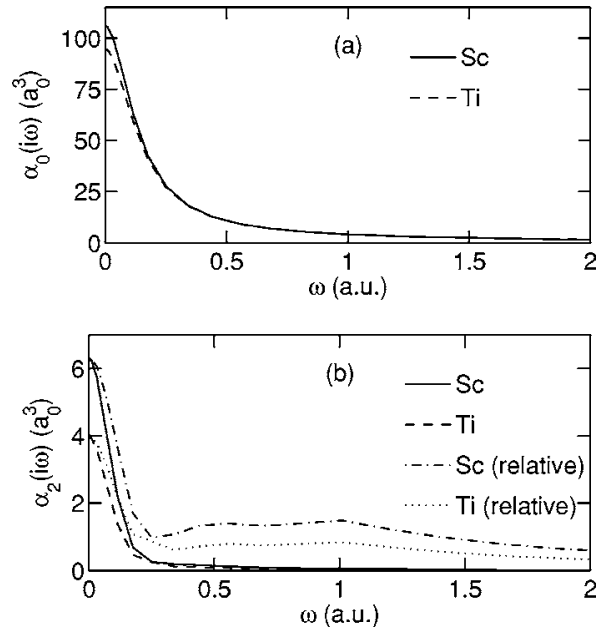

FIG. 2. The dynamic polarizabilities of Sc and Ti at imaginary frequencies. Plot (a) shows the scalar polarizabilities $\alpha_{0}(i \omega)$ for Sc and Ti. Plot (b) shows the tensor polarizabilities $\alpha_{2}(i \omega)$ for Sc (solid line) and $\mathrm{Ti}$ (dashed line) and also the relative tensor polarizabilities $\alpha_{2}(i \omega) / \alpha_{0}(i \omega)$ [multiplied with $\alpha_{0}(0)$ to make it coincide with $\alpha_{2}(i \omega)$ for $\left.\omega=0\right]$ for Sc (dotted-dashed line) and for Ti (dotted line).

abilities $\alpha_{0}(2)$ and $\alpha_{2}(2)$; the root-mean-square deviation is $0.3 \%$, which in an exact theory would be zero. If we were to compute $\alpha_{2}(2)$ from $\alpha_{\|}(2,2)$ and $\alpha_{\perp}(2,2)$ according to Eq. (11) this $0.3 \%$ error would introduce an uncertainty in $\alpha_{2}(2)$ of up to $7 \%$.

In Table I we compare our static polarizabilities with the results of variational configuration interaction calculations by Stiehler and Hinze [17]. They remark that their values are probably too large. Our scalar polarizabilities are indeed smaller, and in better agreement with the variational perturbation calculations of Chandler and Glass [18] and recent calculations by Kłos [5]. The only theoretical tensor polarizabilities that we found are those that can be derived from the $\alpha_{\|}(L, M)$ of Stiehler and Hinze. These agree in sign but they are smaller than ours by $15 \%$ and $45 \%$ for $\mathrm{Sc}$ and $\mathrm{Ti}$, respectively. Our value of 6.31 for $\alpha_{2}$ for Sc is consistent with the experimental value of 6.0(4) of Rinkleff and Thorn [19].

Figure 2 presents the scalar and tensor dynamic polarizabilities of $\mathrm{Sc}$ and $\mathrm{Ti}$ as a function of the imaginary frequency. At each frequency the polarizabilities were obtained from a linear least squares fit. Sometimes the anisotropy in dispersion coefficients is estimated from the relative anisotropy in the static polarizability $\left[\alpha_{2}(L) / \alpha_{0}(L)\right.$, see Eq. (21)] $[20,21]$. Therefore we also show in plot (b) how the relative anisotropy depends on the imaginary frequency. The relative anisotropy for $\omega>0$ is always smaller than for $\omega=0$. For high frequencies it goes to zero, which is expected because both $\alpha_{z z}(i \omega)$ and $\alpha_{x x}(i \omega)$ vary as $N / \omega^{2}$ asymptotically.

In Table II we present the dispersion coefficients. The first four entries are the dispersion coefficients computed from the parallel and perpendicular polarizabilities using Eq. (6). From these results we computed the isotropic and anisotropic dispersion coefficients for Sc using Eq. (17) and for Ti by solving two linear equations [Eq. (20), with $M=2,3$ ] for $C_{6,0}$ and $C_{6,2}$. We also computed the isotropic and anisotropic dispersion coefficients directly from the isotropic and aniso- 
TABLE II. Dispersion coefficients for $\mathrm{He}-\mathrm{Sc}$ and $\mathrm{He}-\mathrm{Ti}$ (in $\left.E_{h} a_{0}^{6}\right)$. We consider the results obtained from Eqs. (18) and (19) to be our most accurate.

\begin{tabular}{llll}
\hline \hline & & $\mathrm{Sc}$ & $\mathrm{Ti}$ \\
\hline$C_{6}(\Sigma)$ & Eq. (6) & 29.56 & \\
$C_{6}(\Pi)$ & Eq. (6) & 29.74 & \\
$C_{6}(\Delta)$ & Eq. (6) & 30.50 & 28.40 \\
$C_{6}(\Phi)$ & Eq. (6) & & 28.68 \\
$C_{6,0}$ & Eqs. (17) and (20) & 30.01 & 28.40 \\
$C_{6,0}$ & Eq. (18) & $\mathbf{3 0 . 0 0}$ & $\mathbf{2 8 . 3 9}$ \\
$C_{6,0}$ & Ref. [5] & 31.4 & 25 \\
$C_{6,2}$ & Eqs. (17) and (20) & -1.71 & -0.84 \\
$C_{6,2}$ & Eq. (19) & $-\mathbf{1 . 6 3}$ & $-\mathbf{0 . 8 9}$ \\
$C_{6,2}$ & Eq. (21) & -3.13 & -1.78 \\
$C_{6,2}$ & Ref. [5] & -0.14 & \\
$C_{6,2}$ & Ref. [20] & -2.39 & \\
$C_{6,2}$ & Ref. [20] & -1.51 & \\
\hline \hline
\end{tabular}

${ }^{\mathrm{a}}$ Estimate based on the relative anisotropy in the static polarizability.

${ }^{\mathrm{b}}$ From a fit to MRCI potentials.

tropic polarizabilities shown in Fig. 2 using Eqs. (18) and (19). The results are very similar. We prefer the method using Eqs. (18) and (19) since it employs scalar and tensor polarizabilities obtained from a linear least squares fit to all computed polarizabilities, which possibly reduces numerical "noise."

Dispersion coefficients for $\mathrm{ScHe}$ and TiHe have been derived by Kłos et al. $[5,20]$ by fitting ab initio potential energy curves and they are included in the table. For the isotropic dispersion interaction in $\mathrm{ScHe}$ the results agree within $5 \%$ and for TiHe the difference is $12 \%$. Kłos et al. remark that the accuracy of their long-range potentials for TiHe was not sufficient to extract isotropic and anisotropic $C_{6}$ coefficients and their result is an estimate. Kłos et al. also give an estimate of $C_{6,2}$ for $\mathrm{ScHe}$ based on the relative anisotropy in the static polarizability which is more than an order of magnitude larger than the result of the fit [5]. When we follow the same procedure [i.e., using Eq. (21)] we find that this overestimates the anisotropy by a factor of 2 for $\mathrm{ScHe}$ as well as TiHe (see Table II). This overestimate can be understood from the frequency dependence of the relative anisotropy shown in Fig. 2.

\section{CONCLUSION}

We have presented isotropic and anisotropic dispersion coefficients for ScHe and TiHe. The coefficients were computed employing dynamic polarizabilities computed with a TDDFT method. The redundancy in the open-shell atomic polarizabilities is used to check the internal consistency of the method and to enhance the accuracy of the computed polarizabilities. We show that a determination of the dispersion anisotropy based on static polarizabilities results in an overestimation by a factor of 2 .

Our calculations confirm earlier estimates that the anisotropy in the long range interaction is small, which suggests that the inelastic rates and trap loss of $\mathrm{Sc}$ and $\mathrm{Ti}$ in a buffer gas cooling experiment will be slow.

\section{ACKNOWLEDGMENTS}

The authors thank Jacek Kłos for sharing his results and Ad van der Avoird for carefully reading the paper. This work is supported by the Chemical Sciences, Geosciences and Biosciences Division of the Office of Basic Energy Sciences, Office of Science, U.S. Department of Energy.

\section{APPENDIX: DERIVATION OF EQS. (8) AND (9)}

The spherical components of the dynamic dipole-dipole polarizability are defined by

$$
\alpha_{q,-q}(L, M ; i \omega)=\sum_{\gamma M_{\gamma}}\left(E_{\gamma}-E_{0}\right) \frac{\left[\left\langle L M\left|\hat{\mu}_{q}\right| \gamma L_{\gamma} M_{\gamma}\right\rangle\left\langle\gamma L_{\gamma} M_{\gamma}\left|\hat{\mu}_{-q}\right| L M\right\rangle+\left\langle L M\left|\hat{\mu}_{-q}\right| \gamma L_{\gamma} M_{\gamma}\right\rangle\left\langle\gamma L_{\gamma} M_{\gamma}\left|\hat{\mu}_{q}\right| L M\right\rangle\right]}{\left(E_{\gamma}-E_{0}\right)^{2}+\omega^{2}},
$$

where the spherical components of the dipole operator are defined by $\hat{\mu}_{0}=\hat{\mu}_{z}$ and $\hat{\mu}_{ \pm}=\mp 2^{-1 / 2}\left(\hat{\mu}_{x} \pm i \hat{\mu}_{y}\right)$. The dipole operator can be expressed in unit spherical tensor operators of rank one [22]

$$
\hat{\mu}_{q}=\frac{1}{\sqrt{3}} \sum_{\gamma_{1} L_{1} \gamma_{2} L_{2}} \hat{T}_{1, q}\left(\gamma_{1} L_{1} ; \gamma_{2} L_{2}\right)\left\langle\gamma_{1} L_{1}\|\hat{\mu}\| \gamma_{2} L_{2}\right\rangle
$$

where

$$
\begin{aligned}
\hat{T}_{k q}\left(\gamma_{1} j_{1} ; \gamma_{2} j_{2}\right)= & \sum_{m_{1} m_{2}}\left|\gamma_{1} j_{1} m_{1}\right\rangle\left\langle\gamma_{2} j_{2} m_{2}\right| \\
& \times(-1)^{j_{1}-m_{1}}[k]^{1 / 2}\left(\begin{array}{ccc}
j_{1} & k & j_{2} \\
-m_{1} & q & m_{2}
\end{array}\right)
\end{aligned}
$$

and $\left\langle\gamma_{1} L_{1}\|\hat{\mu}\| \gamma_{2} L_{2}\right\rangle$ is the reduced matrix element from the Wigner-Eckart theorem. For the spherical tensor operators we have the general relation 


$$
\begin{aligned}
\hat{T}_{k_{1} q_{1}} & \left(\gamma_{1} j_{1} ; \gamma_{2} j_{2}\right) \hat{T}_{k_{2} q_{2}}\left(\gamma_{2} j_{2} ; \gamma_{3} j_{3}\right) \\
= & \sum_{K Q} \hat{T}_{K Q}\left(\gamma_{1} j_{1} ; \gamma_{3} j_{3}\right)\left[k_{1}\right]^{1 / 2}\left[k_{2}\right]^{1 / 2}(-1)^{j_{1}+j_{3}+K} \\
& \times\left\{\begin{array}{lll}
j_{1} & j_{2} & k_{1} \\
k_{2} & K & j_{3}
\end{array}\right\}\left\langle k_{1} q_{1} k_{2} q_{2} \mid K Q\right\rangle .
\end{aligned}
$$

Substituting Eqs. (A2)-(A4) into Eq. (A1) we derive

$$
\begin{aligned}
\alpha_{q,-q}(L, M ; i \omega)= & \alpha_{0}(L ; i \omega) \frac{\langle 1 q 1-q \mid 00\rangle}{\langle 1010 \mid 00\rangle} \\
& +\alpha_{2}(L ; i \omega) \frac{\langle L M 20 \mid L M\rangle\rangle}{\langle L L 20 \mid L L\rangle} \frac{\langle 1 q 1-q \mid 20\rangle}{\langle 1010 \mid 20\rangle},
\end{aligned}
$$

where

$$
\begin{aligned}
\alpha_{K}(L ; i \omega)= & \frac{2[K]^{1 / 2}}{[L]^{1 / 2}} \sum_{\gamma} \frac{\left(E_{\gamma}-E_{0}\right) \mid\left\langle\left. L\|\hat{\mu}\| \gamma L_{\gamma \prime}\right|^{2}\right.}{\left(E_{\gamma}-E_{0}\right)^{2}+\omega^{2}} \\
& \times(-1)^{L+L_{\gamma}}\left\{\begin{array}{ccc}
L & L_{\gamma} & 1 \\
1 & K & L
\end{array}\right\}\langle 1010 \mid K 0\rangle\langle L L K 0 \mid L L\rangle .
\end{aligned}
$$

For $\omega=0$ this expression corresponds to Eq. (19) in Ref. [15] and Eqs. (3.20) and (3.21) in Ref. [14] [remember that $\left.\left\langle L\|\hat{\mu}\| \gamma L_{\gamma}\right\rangle=(-1)^{L-L} \gamma\left\langle\gamma L_{\gamma}\|\hat{\mu}\| L\right\rangle^{*}\right]$. Using $\hat{\mu}_{-q}^{\dagger}=(-1)^{q} \hat{\mu}_{q}$ one readily derives

$$
\begin{gathered}
\alpha_{0,0}(L, M ; i \omega)=\alpha_{\|}(L, M ; i \omega), \\
\alpha_{1,-1}(L, M ; i \omega)=-\alpha_{\perp}(L, M ; i \omega),
\end{gathered}
$$

and by substituting explicit expressions for the ClebschGordan coefficients we can derive Eqs. (8) and (9) from Eq. (A5).
[1] C. I. Hancox, S. C. Doret, M. T. Hummon, L. Luo, and J. M. Doyle, Nature 431, 281 (2004).

[2] C. I. Hancox, S. C. Doret, M. T. Hummon, R. V. Krems, and J. M. Doyle, Phys. Rev. Lett. 94, 013201 (2005).

[3] R. V. Krems, G. C. Groenenboom, and A. Dalgarno, J. Phys. Chem. 108, 8941 (2004).

[4] R. V. Krems, J. Kłos, M. F. Rode, M. M. Szczęśniak, G. Chałasiński, and A. Dalgarno, Phys. Rev. Lett. 94, 013202 (2005).

[5] J. Kłos, M. F. Rode, J. E. Rode, G. Chałasiński, and M. M. Szczęśniak, Eur. Phys. J. D 31, 429 (2004).

[6] X. Chu and A. Dalgarno, J. Chem. Phys. 121, 4083 (2004).

[7] X. Chu and A. Dalgarno, Adv. At., Mol., Opt. Phys. 51, 83 (2005).

[8] J. Callaway and E. Bauer, Phys. Rev. 140, A1072 (1965).

[9] V. Aquilanti and G. Grossi, J. Chem. Phys. 73, 1165 (1980).

[10] R. H. G. Reid and A. Dalgarno, Phys. Rev. Lett. 22, 1029 (1969).

[11] R. H. G. Reid and A. Dalgarno, Chem. Phys. Lett. 6, 85 (1970).
[12] R. H. G. Reid, J. Phys. B 8, 2018 (1973).

[13] A. J. Stone, The Theory of Intermolecular Forces (Oxford University Press, Oxford, 1996).

[14] J. R. P. Angel and P. G. H. Sandars, Proc. R. Soc. London, Ser. A 305, 125 (1968).

[15] A. Khadjavi, A. Lurio, and W. Happer, Phys. Rev. 167, 128 (1968).

[16] M. J. Jamieson, G. W. F. Drake, and A. Dalgarno, Phys. Rev. A 51, 3358 (1995).

[17] J. Stiehler and J. Hinze, J. Phys. B 28, 4055 (1995).

[18] G. S. Chandler and R. Glass, J. Phys. B 20, 1 (1987).

[19] R.-H. Rinkleff and F. Thorn, Z. Phys. D: At., Mol. Clusters 31, 31 (1994).

[20] J. Kłos, J. Chem. Phys. 123, 024308 (2005).

[21] L. Beneventi, P. Casavecchia, and G. G. Volpi, J. Chem. Phys. 85, 7011 (1986).

[22] K. Blum, Density Matrix Theory and Applications (Plenum, New York, 1981). 